\title{
Competition between weak localization and antilocalization in topological surface states
}

\author{
Hai-Zhou Lu, ${ }^{1}$ Junren Shi, ${ }^{2}$ and Shun-Qing Shen ${ }^{1}$ \\ ${ }^{1}$ Department of Physics and Centre of Theoretical and Computational Physics, \\ The University of Hong Kong, Pokfulam Road, Hong Kong, China \\ ${ }^{2}$ International Center for Quantum Materials, Peking University, Beijing 100871, China
}

(Dated: June 2, 2018)

\begin{abstract}
A magnetoconductivity formula is presented for the surface states of a magnetically doped topological insulator. It reveals a competing effect of weak localization and weak antilocalization in quantum transport when an energy gap is opened at the Dirac point by magnetic doping. It is found that, while random magnetic scattering always drives the system from the symplectic to the unitary class, the gap could induce a crossover from weak antilocalization to weak localization, tunable by the Fermi energy or the gap. This crossover presents a unique feature characterizing the surface states of a topological insulator with the gap opened at the Dirac point in the quantum diffusion regime.
\end{abstract}

PACS numbers: 73.25.+i, 03.65.Vf, 73.20.-r, 85.75.-d

Topological surface states, composed of an odd number of massless Dirac cones, are peculiar to threedimensional (3D) topological insulators [1-3]. Electrons in these states have a helical spin structure in momentum space, and acquire a $\pi$ Berry's phase after completing a closed trajectory adiabatically around the Fermi surface. The $\pi$ Berry phase could lead to the absence of backscattering [4], weak antilocalization [5], and the absence of Anderson localization [6, 7]. In the quantum diffusion regime (mean free path $\ll$ system size $\sim$ phase coherent length), an electron maintains its phase coherence after being scattered by static centers for many times. As a result, the destructive interference due to the $\pi$ Berry phase can give a quantum enhancement to the classical electronic conductivity, leading to weak antilocalization (WAL) [8, 9]. Applying a magnetic field tends to break the destructive interference, giving rise to negative magnetoconductivity (MC), a key signature of WAL. WAL is expected in systems with symplectic symmetry. Much effort has been devoted to observing WAL in graphene [5, 10 13]. However, graphene has two valleys of gapless Dirac cones with opposite chiralities, and the intervalley scattering will inevitably suppress WAL [5, 10 13]. In contrast, the surface states of recently discovered topological insulators $\mathrm{Bi}_{2} \mathrm{Te}_{3}$ and $\mathrm{Bi}_{2} \mathrm{Se}_{3}$ have only one helical Dirac cone [14 16], and WAL is intrinsic to them. Many observations of WAL in $\mathrm{Bi}_{2} \mathrm{Te}_{3}$ and $\mathrm{Bi}_{2} \mathrm{Se}_{3}$ have been reported recently $\left.17-22\right]$. In particular, there is great interest in the effect of magnetic doping, which is considered to be an efficient way to open an energy gap in the Dirac cone by breaking time reversal symmetry (TRS) 23 25]. This gap is expected to give rise to many interesting phenomena, such as Majorana fermion 26], topological magnetoelectric effect [27] and quantized anomalous Hall effect [28]. These developments call for a thorough theoretical investigation on WAL in topological insulators, in particular, in the pres- ence of magnetic doping.

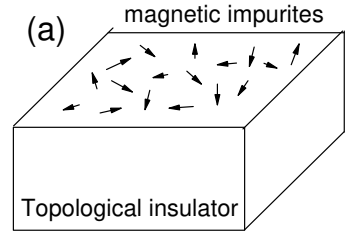

(c)

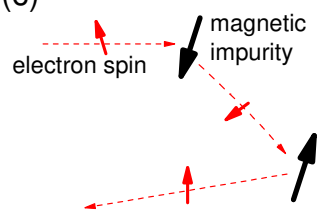

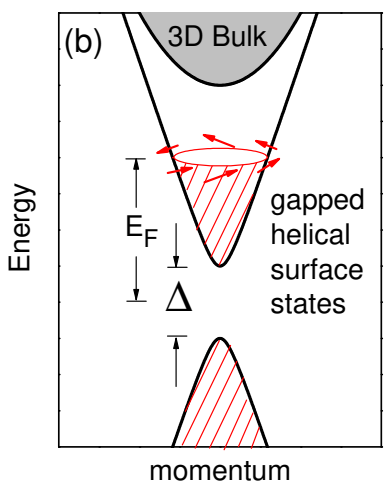

FIG. 1: (a) A topological insulator with magnetic doping on the top surface. (b) Magnetic doping may open a gap $(\Delta)$ at the Dirac point of the surface states [24, 25]. $E_{F}$ is the Fermi energy measured from the Dirac point. $\Delta$ tilts in-plane spin polarization of the massless Dirac fermion out of plane, leading to the deviation from the $\pi$ Berry phase. (c) Scattering of an electron by random magnetic impurities. Dashed lines represent the trajectory of the electron.

In this Letter, a MC formula is presented for the magnetically doped surface states of a topological insulator [Fig. 1(a)]. We assume that the mean field produced by magnetic doping may open a uniform gap at the Dirac point 24, 25] [Fig. 1(b)], and the local fluctuation over the mean field can scatter conducting electrons in a random fashion [Fig. 1(c)]. With the help of the diagrammatic technique [5, 8 10, 29 35], we obtain the MC formula, which consists of two competing terms. Besides the WAL term due to the gapless Dirac fermion, an extra weak localization (WL) term arises as a result of the gap opening. We find that either the gap or the magnetic scattering can drive MC of the system from WAL to a parabolic dependence on the magnetic field $\left(\sim B^{2}\right)$. Fur- 
ther increasing the gap/Fermi energy ratio may drive the system to WL after reaching the $B^{2}$ regime. A crossover from WAL to WL is thus expected, tunable by the size of the TRS-breaking gap or the position of Fermi energy. Beyond the theories for non-Dirac systems with strong spin-orbit coupling [30] or strong ferromagnetism 36], the observation of the crossover will provide an important signature in transport experiments for the existence of a TRS-breaking gap in the topological surface states.

We describe the magnetically doped surface states of a 3D topological insulator by the massive Dirac model $H$ in a random impurity potential $U(\mathbf{r})$. The Hamiltonian of the massive Dirac model is given by

$$
H=\gamma\left(\sigma_{x} k_{y}-\sigma_{y} k_{x}\right)+h_{z} \sigma_{z},
$$

where $\sigma_{x, y, z}$ are the Pauli matrices. $\gamma=\hbar v_{\mathrm{F}}$, with $v_{\mathrm{F}}$ the Fermi velocity. The $\sigma_{z}$ term represents a gap opened by breaking TRS, with $h_{z}=\frac{1}{2} g \mu_{B} B+\frac{\Delta}{2}$, where the first term is the Zeeman energy of the out-of-plane magnetic field $B$, with $g$ the g-factor and $\mu_{B}$ the Bohr magneton. $\Delta$ is the gap opened at the Dirac point 24, 25], it originates from the mean field produced by the magnetic doping. The Hamiltonian describes two energy bands as shown in Fig. 1(b). In this work, we assume that the Fermi energy $E_{F}$ is tuned into the gap of the 3D bulk bands, and intersects with the upper band of the surface states, which is the only band included in the calculation. Its band dispersion is given by $\epsilon_{\mathbf{k}}=\sqrt{\gamma^{2} k^{2}+h_{z}^{2}}$ and the wave function $\psi_{\mathbf{k}}(\mathbf{r})=\left[a,-i e^{i \varphi} b\right]^{T} e^{i \mathbf{k} \cdot \mathbf{r}} / \sqrt{S}$, where $\tan \varphi \equiv k_{y} / k_{x}, \mathbf{k}$ is the wave vector, $a \equiv \cos \frac{\theta}{2}, b \equiv \sin \frac{\theta}{2}$, and $\cos \theta \equiv h_{z} / \sqrt{h_{z}^{2}+\gamma^{2} k^{2}}, S$ is the area. In this work, all the physical quantities will be evaluated at the Fermi energy $E_{F}$ at low temperatures. The density of states at $E_{F}$ is $N_{F}=E_{F} /\left(2 \pi \gamma^{2}\right)$. The scattering by nonmagnetic and magnetic impurities is modeled by the random potential

$$
U(\mathbf{r})=\sum_{i, \alpha} u_{\alpha}^{i} \sigma_{\alpha} \delta\left(\mathbf{r}-\mathbf{R}_{i}\right),
$$

where $\alpha$ runs over $0, x, y, z . \sigma_{0}$ is the $2 \times 2$ unit matrix. $\mathbf{R}_{i}$ are the positions of the randomly distributed impurities. $u_{0}^{i}$ depicts the potential at $\mathbf{R}_{i}$ for nonmagnetic impurity, and $u_{x, y, z}^{i}$ for magnetic impurity. Note that $u_{x, y, z}^{i}$ do not represent the total local exchange field produced by the impurity at $\mathbf{R}_{i}$, but the local fluctuation over the mean field that gives the gap. Therefore, $\langle U(\mathbf{r})\rangle_{\mathrm{imp}}=0$ and we can still assume the random potential is delta-correlated $\left\langle U(\mathbf{r}) U\left(\mathbf{r}^{\prime}\right)\right\rangle_{\mathrm{imp}} \sim \delta\left(\mathbf{r}-\mathbf{r}^{\prime}\right)$, where $\langle\ldots\rangle_{\text {imp }}$ means average over impurity configurations, and we follow the practical assumption that different types of impurity scattering are uncorrelated [10].

The quantum interference correction to conductivity of Dirac fermions can be calculated by the diagrams in Fig. 22 which are different from those for the usual twodimensional electron gas (2DEG) 8, , 9, 31, 32] in several
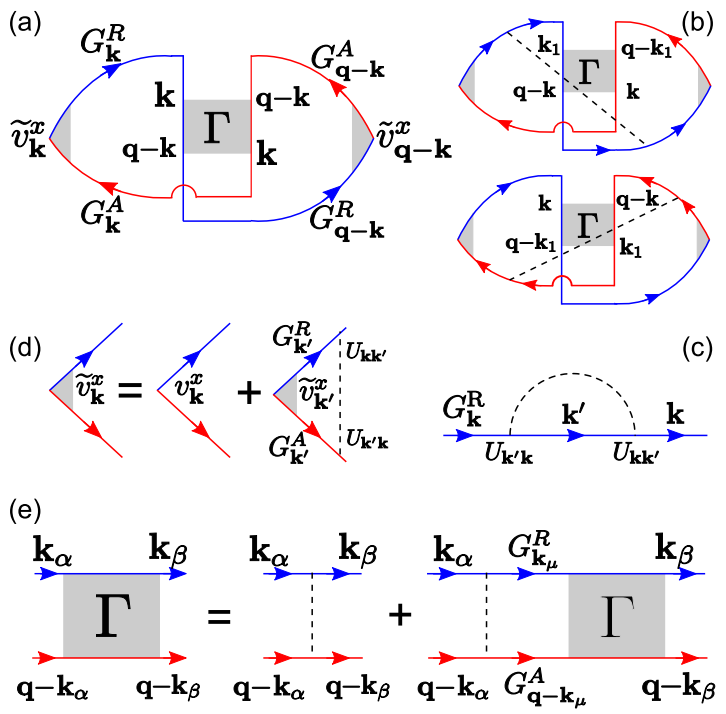

FIG. 2: The diagrams for the quantum interference correction to conductivity of Dirac fermions. The arrowed solid and dashed lines represent the Green's functions and impurity scattering, respectively. (a) The bare [31, 32] and (b) two dressed [10] Hikami boxes give the quantum conductivity correction from the maximally crossed diagrams. (c) The retarded Green function with the first-order Born approximation to the impurity-averaged self-energy. (d) Ladder diagram vertex correction to velocity 29]. (e) The Bethe-Salpeter equation for the vertex of maximally crossed diagrams.

aspects [5, 10, 29]. (i) Besides the conventional maximally crossed diagram (bare Hikami box) in Fig. 2(a), two dressed Hikami boxes in Fig. 2(b) are also needed; each gives $-1 / 4$ as the bare Hikami box for the gapless Dirac cone. (ii) The ladder diagram correction to the bare velocity $v_{\mathbf{k}}^{x} \equiv(1 / \hbar) \partial \epsilon_{\mathbf{k}} / \partial k_{x}$ [Fig. 2(d)] must be taken into account, which corrects the velocity to $\widetilde{v}_{\mathbf{k}}^{x}=2 v_{\mathbf{k}}^{x}$ for the gapless Dirac cone. We generalize these conclusions for the gapless case to the gapped Dirac cone as follows.

The arrowed lines in Fig. 2 stand for the impurityaveraged retarded (R) and advanced (A) Green's functions $G_{\mathbf{k}}^{\mathrm{R} / \mathrm{A}}(\omega)=1 /\left(\omega-\epsilon_{\mathbf{k}} \pm i \hbar / 2 \tau\right)$, where under the firstorder Born approximation, the impurity-induced selfenergy is given by the total scattering time $\tau$, with $\hbar / \tau \equiv 2 \pi \sum_{\mathbf{k}^{\prime}}\left\langle\left|U_{\mathbf{k}^{\prime} \mathbf{k}}\right|^{2}\right\rangle_{\mathrm{imp}} \delta\left(\omega-\epsilon_{\mathbf{k}^{\prime}}\right)$, where $U_{\mathbf{k}, \mathbf{k}^{\prime}} \equiv$ $\left\langle\psi_{\mathbf{k}}(\mathbf{r})|U(\mathbf{r})| \psi_{\mathbf{k}^{\prime}}(\mathbf{r})\right\rangle$ is the scattering amplitude between two momenta. It can be separated into $1 / \tau=1 / \tau_{e}+$ $1 / \tau_{m}$, where the nonmagnetic elastic scattering time $\tau_{e}$ is given by $\hbar / \tau_{e}=2 \pi N_{F} n_{0} u_{0}^{2}\left(a^{4}+b^{4}\right)$ and the total magnetic scattering time $\tau_{m}$ can be separated into $1 / \tau_{m}=2 / \tau_{x}+1 / \tau_{z}$, with $\hbar / \tau_{z}=2 \pi N_{F} n_{m} u_{z}^{2}\left(a^{4}+b^{4}\right)$ and $\hbar / \tau_{x}=2 \pi N_{F} n_{m} u_{x}^{2}\left(2 a^{2} b^{2}\right)$. In-plane isotropy $\left(u_{x}=u_{y}\right)$ is assumed. $u_{0}$ depicts the average scattering strength for nonmagnetic impurities, while $u_{x, y, z}$ for magnetic impurities. $n_{0}$ and $n_{m}$ are concentrations of nonmagnetic and magnetic impurities, respectively. $\tau_{e}$ and $\tau_{m}$ are related 
to the elastic scattering length $\ell_{e}$ and magnetic scattering length $\ell_{m}$ by $\ell_{e}=\sqrt{D \tau_{e}}$ and $\ell_{m}=\sqrt{D \tau_{m}}$, respectively. $D \equiv v_{F}^{2} \tau^{2} / 2$ is the diffusion constant. Considering the poor surface mobility 18 20], we assume that $\ell_{e}$ is much shorter than the phase coherence length $\ell_{\phi}$, as required by the quantum diffusion transport.

In the conductivity diagrams Figs. 2(a) and (b), the vertex function $\Gamma$ from the maximally crossed diagrams usually is proportional to $1 / q^{2}$, where $\mathbf{q}$ is the summation of momenta before and after scattering. Since $\Gamma$ diverges as $q \rightarrow 0$, it contributes mainly to backscattering. This allows us to sum $\mathbf{k}$ and $\mathbf{k}_{1}$ for small $q$ first, and write the zero-temperature conductivity correction from the bare and two dressed Hikami boxes as

$$
\sigma^{F}=-\frac{e^{2} N_{F} v_{F}^{2} \tau^{3} \sin ^{2} \theta}{\hbar^{2}} \eta_{v}^{2}\left(1+2 \eta_{H}\right) \sum_{\mathbf{q}} \Gamma(\mathbf{q}),
$$

where $\eta_{v}$ comes from the correction to velocity from the ladder diagrams in Fig. 2(d), with

$$
\eta_{v}=\left[1-\frac{1}{2}\left(\frac{\tau}{\tau_{e}}-\frac{\tau}{\tau_{z}}\right) \frac{2 a^{2} b^{2}}{a^{4}+b^{4}}\right]^{-1},
$$

and each of the dressed Hikami boxes gives an extra $\eta_{H}$ contribution as the bare Hikami box, with

$$
\eta_{H}=-\frac{1}{2}\left(1-\eta_{v}^{-1}-\frac{\tau}{\tau_{x}}\right) .
$$

$\eta_{v}$ and $\eta_{H}$ reduce to 2 and $-1 / 4$, respectively, in the absence of magnetic doping [5, 10, 29].

In Fig. 2, the total momentum conserves on the incoming and outgoing sides of the vertex $\Gamma$, allowing the Bethe-Salpeter equation of the vertex to be written as [5] $\Gamma_{\mathbf{k}_{\alpha} \mathbf{k}_{\beta}}=\Gamma_{\mathbf{k}_{\alpha} \mathbf{k}_{\beta}}^{0}+\sum_{\mathbf{k}_{\mu}} \Gamma_{\mathbf{k}_{\alpha} \mathbf{k}_{\mu}}^{0} G_{\mathbf{k}_{\mu}}^{R} G_{\mathbf{q}-\mathbf{k}_{\mu}}^{A} \Gamma_{\mathbf{k}_{\mu} \mathbf{k}_{\beta}}$, where $\mathbf{k}_{\alpha}+\mathbf{k}_{\beta}=\mathbf{q}, \mathbf{k}_{\alpha, \beta}$ are the incoming and outgoing momenta, respectively. For small $q$, the bare vertex $\Gamma_{\mathbf{k}_{\alpha} \mathbf{k}_{\beta}}^{0} \equiv$ $\left\langle U_{\mathbf{k}_{\beta}, \mathbf{k}_{\alpha}} U_{\mathbf{q}-\mathbf{k}_{\beta}, \mathbf{q}-\mathbf{k}_{\alpha}}\right\rangle_{\mathrm{imp}}$ is found as $\Gamma_{\mathbf{k}_{\alpha} \mathbf{k}_{\beta}}^{0} \approx \frac{\hbar}{2 \pi N_{F}}[A+$ $\left.B e^{i\left(\varphi_{\alpha}-\varphi_{\beta}\right)}+C e^{i 2\left(\varphi_{\alpha}-\varphi_{\beta}\right)}\right]$, with $A=\left(\tau_{e}^{-1}+\tau_{z}^{-1}\right) \frac{a^{4}}{a^{4}+b^{4}}$, $B=\left[\left(\tau_{e}^{-1}-\tau_{z}^{-1}\right) \frac{2 a^{2} b^{2}}{\left(a^{4}+b^{4}\right)}-2 \tau_{x}^{-1}\right], C=\left(\tau_{e}^{-1}+\tau_{z}^{-1}\right) \frac{b^{4}}{a^{4}+b^{4}}$. Different from the usual 2DEG, both the bare vertex $\Gamma_{\mathbf{k}_{\alpha} \mathbf{k}_{\beta}}^{0}$ and the advanced Green function $G_{\mathbf{q}-\mathbf{k}_{\mu}}^{A}$ are explicit functions of the momentum angle. We propose an ansatz to the full vertex function

$$
\Gamma_{\mathbf{k}_{\alpha} \mathbf{k}_{\beta}}=\frac{\hbar}{2 \pi N_{F} \tau} \sum_{n, m \in 0,1,2} \gamma_{n m} e^{i\left(n \varphi_{\alpha}-m \varphi_{\beta}\right)},
$$

where $\gamma_{n m}$ are the expansion coefficients independent of $\varphi_{\alpha, \beta}$. By putting the ansatz into the Bethe-Salpeter equation and expanding $G_{\mathbf{q}-\mathbf{k}_{\mu}}^{A}$ up to $q^{2}$, we obtain the solution to the expansion coefficients

$$
\gamma=2\left[\begin{array}{ccc}
g_{0}+Q^{2} & i Q_{+} & \frac{1}{2} Q_{+}^{2} \\
i Q_{-} & g_{1}+Q^{2} & i Q_{+} \\
\frac{1}{2} Q_{-}^{2} & i Q_{-} & g_{2}+Q^{2}
\end{array}\right]^{-1}
$$

where $Q_{ \pm}=Q_{x} \pm i Q_{y}, Q^{2}=Q_{x}^{2}+Q_{y}^{2}, \mathbf{Q}=v_{F} \tau \sin \theta\left(q_{x}, q_{y}\right)$, and the "Cooperon gaps" $g_{0} \equiv 2\left[\frac{a^{4}+b^{4}}{a^{4}} \frac{1 / \tau}{\left(1 / \tau_{e}+1 / \tau_{z}\right)}-1\right]$, $g_{1} \equiv 2\left[\frac{1 / \tau}{\left(1 / \tau_{e}-1 / \tau_{z}\right) \frac{2 a^{2} b^{2}}{a^{4}+b^{4}}-2 / \tau_{x}}-1\right], \quad g_{2} \equiv$ $2\left[\frac{a^{4}+b^{4}}{b^{4}} \frac{1 / \tau}{\left(1 / \tau_{e}+1 / \tau_{z}\right)}-1\right]$. We note that it is crucial to include all the off-diagonal terms of $\gamma$ in the calculation. Without them, the vertex will be 2 times larger [33, 34] when going back to the gapless limit [5], and the derived $\mathrm{MC}$ formula can not recover to that for $1 / 4$ of graphene [10]. $\Gamma(\mathbf{q})$ in Eq. (3) can be obtained by letting $\mathbf{k}_{\alpha}=\mathbf{k}$ and $\mathbf{k}_{\beta}=\mathbf{q}-\mathbf{k}$ in $\Gamma_{\mathbf{k}_{\alpha} \mathbf{k}_{\beta}}$, and for $q \rightarrow 0$, $\varphi_{\mathbf{k}}-\varphi_{\mathbf{q}-\mathbf{k}} \approx \pi$. Finally, we collect the most divergent terms of the vertex

$$
\Gamma(\mathbf{q}) \approx \frac{\hbar /\left(\pi N_{F} \tau\right)}{g_{0}+\left(1+\frac{1}{g_{1}}\right) Q^{2}}-\frac{\hbar /\left(\pi N_{F} \tau\right)}{g_{1}+\left(1+\frac{1}{g_{0}}+\frac{1}{g_{2}}\right) Q^{2}}
$$
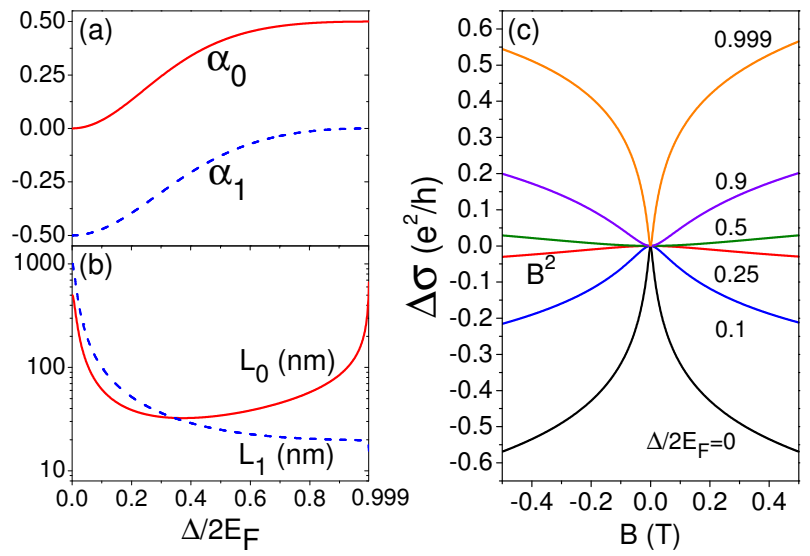

FIG. 3: (a) WL $\left(\alpha_{0}\right)$ and WAL $\left(\alpha_{1}\right)$ weight factors as functions of $\Delta / 2 E_{F}$, where $\Delta$ is the gap, $E_{F}$ is the Fermi energy. (b) WL $\left(\ell_{0}\right)$ and WAL $\left(\ell_{1}\right)$ lengths as functions of $\Delta / 2 E_{F}$. (c) Magnetoconductivity $\Delta \sigma(B)$ for different $\Delta / 2 E_{F}$ in the limit of weak magnetic scattering. $\ell_{\phi}=300 \mathrm{~nm}$. $\ell_{m}=1000$ nm. $u_{x}=u_{z}$ is assumed.

Zero-field conductivity correction $\sigma^{F}(0)$ can be calculated by performing the integral over $q$ in Eq. (3) between $1 / \ell_{e}$ and $1 / \ell_{\phi}$, respectively [5]. In the presence of the perpendicular magnetic field $B, q^{2}$ will be quantized into $q_{n}^{2}=(n+1 / 2)(4 e B / \hbar) \equiv(n+1 / 2) / \ell_{B}^{2}$, where $n$ labels the Landau levels. Summation over $n$ gives the conductivity correction $\sigma^{F}(B)$ at finite field [8]. The magnetoconductivity $\Delta \sigma(B) \equiv \sigma^{F}(B)-\sigma^{F}(0)$ is found for $\ell_{B}^{2} / \ell_{e}^{2} \gg 1$ as

$$
\Delta \sigma(B)=\sum_{i=0,1} \frac{\alpha_{i} e^{2}}{\pi h}\left[\Psi\left(\frac{\ell_{B}^{2}}{\ell_{\phi}^{2}}+\frac{\ell_{B}^{2}}{\ell_{i}^{2}}+\frac{1}{2}\right)-\ln \left(\frac{\ell_{B}^{2}}{\ell_{\phi}^{2}}+\frac{\ell_{B}^{2}}{\ell_{i}^{2}}\right)\right],
$$


with $\Psi$ the digamma function,

$$
\begin{aligned}
& \alpha_{1}=-\frac{\eta_{v}^{2}\left(1+2 \eta_{H}\right)}{2\left(1+\frac{1}{g_{0}}+\frac{1}{g_{2}}\right)}, \ell_{1}^{-2}=\frac{g_{1}}{2 \ell^{2} \sin ^{2} \theta\left(1+\frac{1}{g_{0}}+\frac{1}{g_{2}}\right)}, \\
& \alpha_{0}=\frac{\eta_{v}^{2}\left(1+2 \eta_{H}\right)}{2\left(\frac{1}{g_{1}}+1\right)}, \ell_{0}^{-2}=\frac{g_{0}}{2 \ell^{2} \sin ^{2} \theta\left(\frac{1}{g_{1}}+1\right)},
\end{aligned}
$$

and $1 / \ell^{2} \equiv 1 / \ell_{e}^{2}+1 / \ell_{m}^{2}$. In the absence of magnetic impurities, $\alpha_{0}=0, \alpha_{1}=-1 / 2$, one predicts WAL with a prefactor $-1 / 2$, consistent with the experimental observations [18 20]. For a finite gap, because $\alpha_{0}$ and $\alpha_{1}$ have opposite signs, the $\mathrm{MC}$ formula has two competing contributions, $\alpha_{1}$ leads to WAL, $\alpha_{0}$ to WL. $\ell_{0}$ and $\ell_{1}$ give corrections to $\ell_{\phi}$, in particular, when they are much shorter than $\ell_{\phi}$. This formula is the key result of this work.

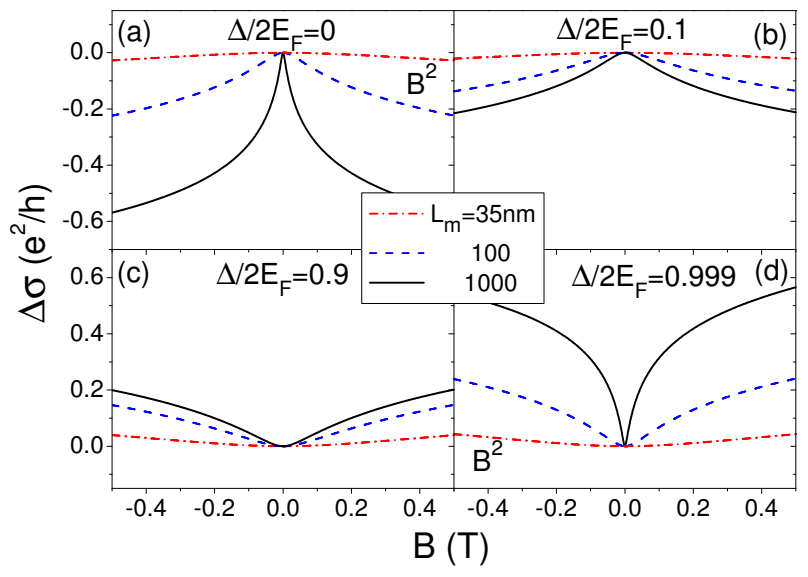

FIG. 4: Magnetoconductivity $\Delta \sigma(B)$ for different magnetic scattering lengths $\ell_{m}$ and $\Delta / 2 E_{F} . \ell_{\phi}=300 \mathrm{~nm} . u_{x}=u_{z}$ is assumed. Shorter $\ell_{m}$ means stronger magnetic scattering.

We first examine the limit of weak magnetic scattering, i.e., $\ell_{m} \gg \ell_{\phi}[17-20]$. We plot MC for different $\Delta / 2 E_{F}$ in Fig. 33(c). For $\Delta / 2 E_{F}=0, \mathrm{MC}$ shows a positive cusp, which is the signature of WAL. As $\Delta / 2 E_{F}$ increases, MC gradually develops a $B^{2}$ dependence, and the system evolves into the unitary regime. Further increasing $\Delta / 2 E_{F}$ will change the sign of $\mathrm{MC}$ from negative to positive, i.e., a WL-like MC. Different from the usual 2DEG, the WL-like MC here has a prefactor $\sim 1 / 2$, instead of 1 31]. This can be seen in Fig. 3(a), where we show the weight factors of the competing WL and WAL terms in the MC formula. In the limit of small $\Delta / 2 E_{F}$, $\alpha_{1}$ overweighs $\alpha_{0}$, so MC is mainly contributed by WAL, with the maximal prefactor $-1 / 2$. In the limit of large $\Delta / 2 E_{F}, \alpha_{1}$ vanishes and $\alpha_{0}$ goes to $1 / 2$, then we have WL with the maximal prefactor $1 / 2$. As shown in Fig. 3(b), either $\ell_{1}$ for small $\Delta / 2 E_{F}$ or $\ell_{0}$ for large $\Delta / 2 E_{F}$ is much larger than $\ell_{\phi}$, this keeps the system well inside the quantum diffusion regime, and protects WAL or WL. For intermediate $\Delta / 2 E_{F}$, where both the WL and
WAL terms contribute, the weak $B^{2}$ MC indicates that the system is driven from the quantum to classical diffusion regime due to the effective reduction of $\ell_{\phi}$ by the much shorter $\ell_{0}$ and $\ell_{1}$. The crossover from WAL to WL by changing $\Delta / 2 E_{F}$ can be understood with the Berry phase 4], which is readily evaluated for the surface band $\psi_{\mathbf{k}}(\mathbf{r})$ as

$$
-i \int_{0}^{2 \pi} d \varphi\left\langle\psi_{\mathbf{k}}(\mathbf{r}) \mid \frac{\partial}{\partial \varphi} \psi_{\mathbf{k}}(\mathbf{r})\right\rangle=\pi\left(1+\frac{\Delta}{2 E_{F}}\right) .
$$

It gives $\pi$ for WAL when $\Delta / 2 E_{F}=0$, and $2 \pi$ for WL when $\Delta=2 E_{F}$. A similar argument was also given for the gap opened by the finite size effect [37]. In the limit of strong magnetic scattering $\ell_{m} \ll \ell_{\phi}$, both WAL and WL are suppressed, as shown in Fig. 4. On the other hand, because the Fermi energy in the ratio $\Delta / E_{F}$ can be controlled independently by gate voltage [18, 19], it is possible to observe the transition from negative to positive MC by tuning the gate voltage even in this limit.

We thank H. T. He, J. N. Wang, F. C. Zhang, W. Q. Chen, X. Dai, and B. Zhou for helpful discussions. HaiZhou also thank K. Imura and H. Suzuura for stimulating discussions. This work is supported by the Research Grant Council of Hong Kong under Grant No. HKU 7051/10P and HKUST3/CRF/09. Junren is supported by NSFC No. 10734110 and 973 program of China No. 2009 CB929101.

[1] M. Z. Hasan and C. L. Kane, Rev. Mod. Phys. 82, 3045 (2010).

[2] X. L. Qi and S. C. Zhang, arXiv:1008.2026 (2010).

[3] J. E. Moore, Nature 464, 194 (2010).

[4] T. Ando, T. Nakanishi, and R. Saito, J. Phys. Soc. J. 67, 2857 (1998).

[5] H. Suzuura and T. Ando, Phys. Rev. Lett. 89, 266603 (2002).

[6] J. H. Bardarson, J. Tworzydło, P. W. Brouwer, and C. W. J. Beenakker, Phys. Rev. Lett. 99, 106801 (2007).

[7] K. Nomura, M. Koshino, and S. Ryu, Phys. Rev. Lett. 99, 146806 (2007).

[8] G. Bergmann, Phys. Rep. 107, 1 (1984).

[9] P. A. Lee and T. V. Ramakrishnan, Rev. Mod. Phys. 57, 287 (1985).

[10] E. McCann, et al., Phys. Rev. Lett. 97, 146805 (2006).

[11] X. Wu, X. Li, Z. Song, C. Berger, and W. A. de Heer, Phys. Rev. Lett. 98, 136801 (2007).

[12] R. V. Gorbachev, F. V. Tikhonenko, A. S. Mayorov, D. W. Horsell, and A. K. Savchenko, Phys. Rev. Lett. 98, 176805 (2007).

[13] F. V. Tikhonenko, A. A. Kozikov, A. K. Savchenko, and R. V. Gorbachev, Phys. Rev. Lett. 103, 226801 (2009).

[14] Y. Xia, et al., Nat. Phys. 5, 398 (2009).

[15] H. J. Zhang, et al., Nat. Phys. 5, 438 (2009).

[16] Y. L. Chen, et al., Science 325, 178 (2009).

[17] H. Peng, et al., Nat. Mater. 9, 225 (2010).

[18] J. Chen, et al., Phys. Rev. Lett. 105, 176602 (2010). 
[19] J. G. Checkelsky, et al., arXiv:1003.3883 (2010).

[20] H. T. He, et al., Phys. Rev. Lett. 106, 166805 (2011).

[21] M. Liu, C. Z. Chang, Z. Zhang, Y. Zhang, W. Ruan, K. He, L. L. Wang, X. Chen, J. F. Jia, S. C. Zhang, Q. K. Xue, X. Ma, Y. Wang, Phys. Rev. B 83, 165440 (2011).

[22] J. Wang, A. M. DaSilva, C. Z. Chang, K. He, J. K. Jain, N. Samarth, X. C. Ma, Q. K. Xue, M. H. W. Chan, Phys. RevB. 83245438 (2011).

[23] Y. S. Hor, et al., Phys. Rev. B 81, 195203 (2010).

[24] Y. L. Chen, et al., Science 329, 659 (2010).

[25] L. A. Wray, et al., Nat. Phys. 7, 32 (2011).

[26] L. Fu and C. L. Kane, Phys. Rev. Lett. 102, 216403 (2009); A. R. Akhmerov, J. Nilsson, and C. W. J. Beenakker, ibid 102, 216404 (2009); K. T. Law, and P. A. Lee, and T. K. Ng, ibid 103, 237001 (2009).

[27] X. L. Qi, T. L. Hughes, S. C. Zhang, Phys. Rev. B 78, 195424 (2008).

[28] R. Yu, et al., Science 329, 61 (2010).

[29] N. H. Shon and T. Ando, J. Phys. Soc. Jpn. 67, 2421
(1998).

[30] S. V. Iordanskii, Yu. B. Lyanda-Geller, G. E. Pikus, JETP Lett. 60, 206 (1994).

[31] S. Hikami, A. I. Larkin, and Y. Nagaoka, Prog. Theor. Phys. 63, 707 (1980).

[32] B. L. Altshuler, D. Khmelnitzkii, A. I. Larkin, and P. A. Lee, Phys. Rev. B 22, 5142 (1980).

[33] K. I. Imura, Y. Kuramoto, and K. Nomura, Phys. Rev. B 80, 085119 (2009).

[34] K. I. Imura, Y. Kuramoto, and K. Nomura, EPL 89, 17009 (2010).

[35] X. Z. Yan and C. S. Ting, Phys. Rev. Lett. 101, 126801 (2008)

[36] V. K. Dugaev, P. Bruno, and J. Barnaś, Phys. Rev. B 64, 144423 (2001).

[37] P. Ghaemi, R. S. K. Mong, and J. E. Moore, Phys. Rev. Lett. 105, 166603 (2010). 